\title{
Real-Time Path Loss Modelling for a More Robust Wireless Performance
}

\author{
Q. Braet ${ }^{1}$, D. Plets ${ }^{1}$, W. Joseph ${ }^{1}$, L. Martens ${ }^{1}$ \\ ${ }^{1}$ Information Technology Department, Ghent University/iMinds \\ Gaston Crommenlaan 8, B-9050 Ghent, Belgium \\ quentin.braet@intec.ugent.be
}

\begin{abstract}
The use of wireless communication systems is important for next-generation industrial environments. To be able to set up a robust network that reacts to changes in the environment, a system for real-time updating path loss models is introduced, based on a continuous measurement of the signal strength in the network. The system is a necessary building block for the creation of a fully automated wireless network planner.
\end{abstract}

Index Terms-propagation, measurement, WiFi, cognitive, path loss model

\section{INTRODUCTION}

The use of wireless communication systems is important to automate and streamline production processes of industrial manufacturing centres, and to facilitate stock monitoring in their warehouses. However, integration of modern wireless communication systems in industrial environments is very challenging and complex: it requires high stability, robustness and reaction speed.

Until now, research was mainly focused on propagation in static office environments [1], only a few publications exist on modelling in industrial environments [2], but these don't integrate automated measurements and path loss model updates in real time, although this is indispensable in this type of environment. There will be humans and other moving objects in the environment and due to the presence of highly reflective materials such as metal, it is not always easy to predict the coverage of the wireless signal. One also has to keep in mind that the Quality of Service (QoS) will sometimes change because of the obstacles being replaced, e.g., in a warehouse. To know how to react to these environmental changes, one has to know how the signal strength distribution is changing. Therefore, this research will focus on the development of a real-time path loss calculation module that uses mobile robots and monitoring probes for estimating the best path loss model in the considered environment. These models can then be used to adapt the wireless network configuration in real time.

Section II will discuss the methodology of the measurement system. In Section III experiments inside the testbed are discussed. Section IV discusses experiments inside an office environment and the integration with an existing network planner. Final remarks and future work are discussed in Section V.

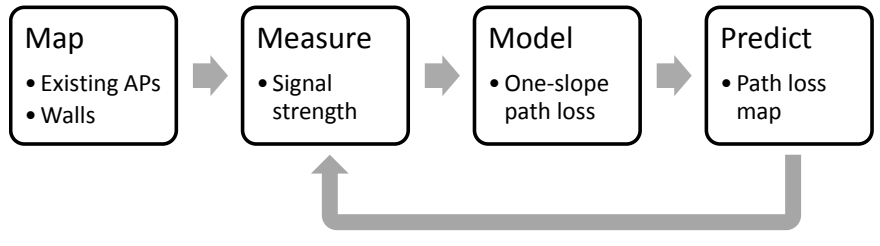

Fig. 1. Methodology overview

\section{Methodology}

The wireless optimization process consists of different phases illustrated in Fig. 1. Firstly, a map of the environment is created, with all the walls and existing access points and nodes. This map will be used by the network planner to visualize the resulting coverage map and will be used as a guide for the robot. Secondly, signal strength measurements are executed. This can be done with fixed nodes (Section III-A), as well as with a mobile robot node that can drive freely through the environment (Section III-B). To minimize interference, only one access point is enabled at a time while executing the experiments. Thirldy, these measurements are used to build a one-slope path loss model. Fourthly, this model, together with the floor plan, will serve as input to the network planner (WHIPP tool [1]). The tool predicts the coverage in the considered environment with the existing access points. The process is repeated by continuously executing measurements to update the path loss model when the environment changes. When needed, it is able to give suggestions about where to place new access points.

\section{A. Test Environments}

1) Testlab W-iLab.t: The first experiments are conducted in a pseudo-shielded testbed environment [3] in Ghent, Belgium. The nodes in the testbed are mounted in an open room (66 $\mathrm{m} \times 20.5 \mathrm{~m}$ ) in a grid configuration with an x-separation of 6 $\mathrm{m}$ and a y-separation of $3.6 \mathrm{~m}$. Fig. 2 shows the ground plan of the testbed with an indication of the location of the nodes. The 60 installed nodes are represented by the blue dots on the picture. Each node has two WiFi interfaces (Sparklan WPEA110N/E/11n mini PCIe 2T2R chipset: AR9280) and on each WiFi card, two antennas are connected (2x2 MIMO (MultipleInput Multiple-Output) is supported). Furthermore, an RM090 sensor node and a USB 2.0 Bluetooth interface (Micro CI2 - 


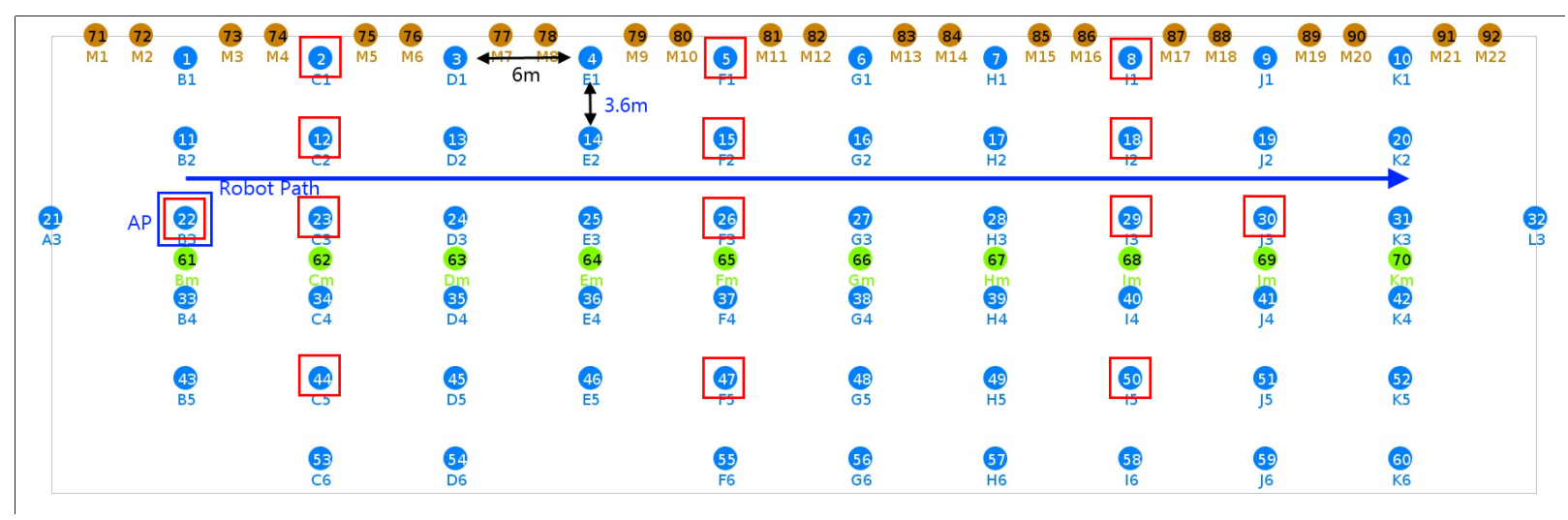

Fig. 2. Overview of the w.iLab-t topology. The blue dots indicate the static Zotac nodes in the testbed. Only the blue nodes with a red square around it, are used in our experiments. The node on the left with two squares is used as access point. The blue arrow indicates the path along which the robot performed measurements.

v3.0 EDR) are incorporated into each node. We consider here experiments using $802.11 \mathrm{~g} / \mathrm{n} \mathrm{WiFi}$ at $2.4 \mathrm{GHz}$. There also are two robots available for experiments in the testbed, a more thorough description of the robots can be found in Section II-B.

2) Office Environment: The goal of the system is to use it outside of the testbed. Therefore, another experiment is executed in a corridor on the third floor of the Zuiderpoort office building in Ghent. The building is a modern three-storey building, with movable walls (layered drywalls) around a core consisting of concrete walls. Figure 3 shows a part of the map of the third floor where the experiment is performed. The signal strength of a D-Link DI-624+ access point, mounted on a cardboard box at a height of $45 \mathrm{~cm}$, is measured.

\section{B. Mobile Robot}

The node used for mobile measurements is of the same type as the static nodes in the testbed. The node is mounted on top of an iRobot Roomba vacuum cleaner that has been modified with a custom circuit board and ZigBee connectivity. An extra battery pack was installed to power the Zotac ZBox node, a compact and energy friendly PC [4]. This node works completely independent of the robot control mechanism. Fig. 4 shows a picture of the robot with a description of its components. In Figure 5, the tasks for each component are shown: the circuit board takes control of the robot movements and its location, the Zotac node performs the wireless measurements.

The exact position of the robots is known at all times: a dead reckoning algorithm is implemented that uses the internal Roomba logic to measure the speed and angle of the robot. Using a taped grid on the floor, corrections can be made to the location algorithm. There are three reasons why it is interesting to do these measurements with a robot. Firstly, when performing measurements, there is often influence of nearby people. Secondly, manually performing a measurement and moving to the next position is time-consuming. This process can be executed a lot faster with a robot. Thirdly, the reproducibility of the experiments is much better.

\section{Path loss model}

The path loss model used in this work, is a one-slope distance-dependent function:

$$
P L=P L_{0}+10 * n * \log _{10}\left(\frac{d}{d_{0}}\right)+X_{\sigma}
$$

In (1), $P L_{0}[\mathrm{~dB}]$ is the path loss at an arbitrarily chosen reference distance $d_{0}[\mathrm{~m}]$, chosen at $1 \mathrm{~m}$ here, $n[-]$ is the path loss exponent. $X_{\sigma}[\mathrm{dB}]$ is a lognormally distributed random variable with zero median (expressed in $\mathrm{dB}$ ) and standard deviation $\sigma[\mathrm{dB}]$. The linear least-squares method is used to determine the optimal values for $n$ and $P L_{0}$ when fitting this model to a set of measurements.

The $95 \%$ shadowing margin of the model is determined by multiplying 1.7 with the standard deviation of the measured PL values around the fitted model [2][5].

\section{Implementation}

The main modules of the application are shown in Fig. 5. All experiments are controlled by the central server framework. This framework can control the robot movements and perform measurements with the Zotac nodes on the robot, or the fixed Zotac nodes in the testbed. Figure 6 shows a sequence diagram with an overview of how all the modules work together. In the next sections, the control framework, the nodes and path loss module are discussed in more detail.

1) The control framework: The core of the system is a central Java application that is run on a laptop. This laptop has to be able to connect to the nodes (either via WiFi or a wired network) to initiate the measuring processes. It is also this framework that initiates the WHIPP tool coverage calculations and planning algorithms with the variables derived from the measurements.

The movements of the robot are also controlled by the framework. A ZigBee interface is required to allow the framework to communicate with the Roomba circuit board.

The sequence diagram in Fig. 6, shows how the experiment controller steers the robot and commands the Zotac node to do the measurements. 


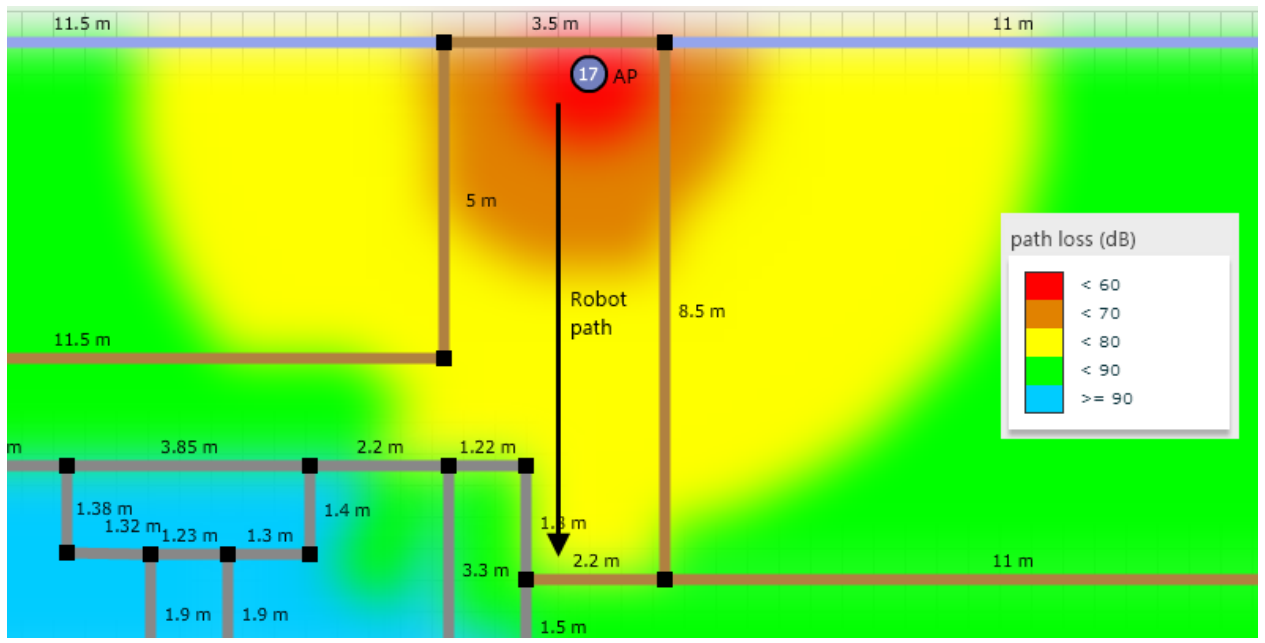

Fig. 3. Path loss map of the corridor, built in real time based on the robot measurements. The black arrow indicates the path where the robot performed measurements. The blue circle indicates the location of the access point and its EIRP (17dBm).

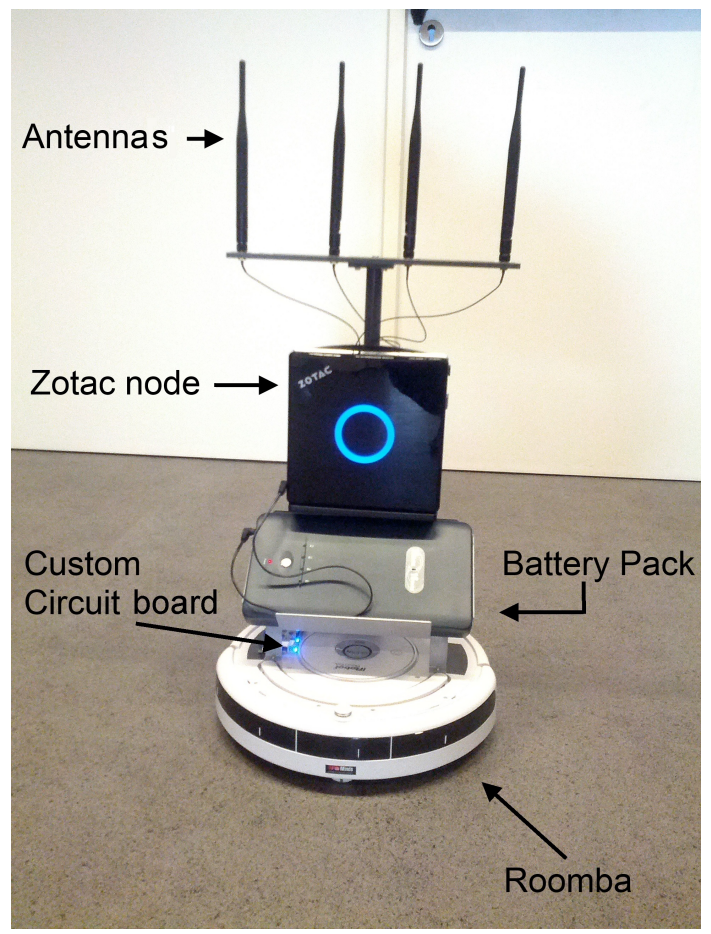

Fig. 4. The robot used for the measurements. The antenna's are placed on a height of $70 \mathrm{~cm}$.

2) The nodes: the nodes run on an Ubuntu distribution. The signal strength $[\mathrm{dBm}]$ is measured with the Wavemon tool [6], a monitoring application for WiFi interfaces on the Linux operating system. It is installed on every node used in the experiments, together with Ruby scripts to control the experiment over a SSH (Secure Shell) connection. As shown in Fig. 6, the results of those measurements are stored in the central database, and can be used by the control framework. The wavemon tool can read the signal strength of the currently connected network every $10 \mathrm{~ms}$.

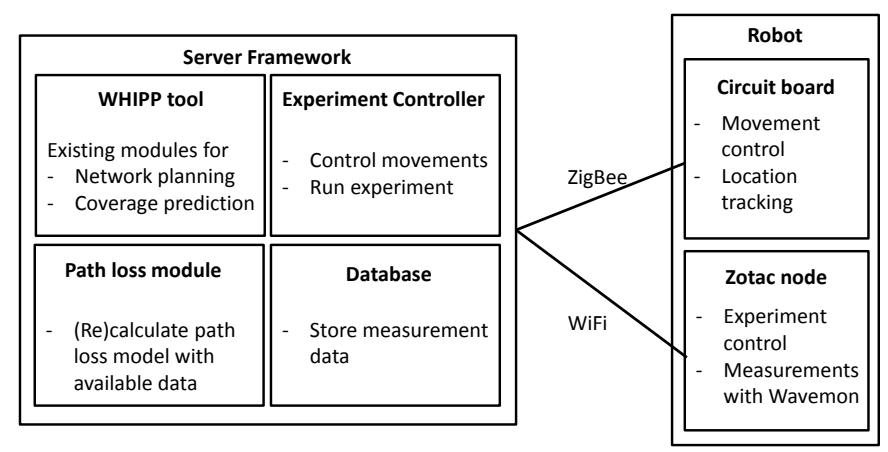

Fig. 5. Main building blocks of the application

3) Updating the path loss model: While the experiments are running, path loss samples are continuously recorded, according to the link-budget formula in (2).

$$
P L[d B]=P_{T}[d B m]+G_{T}[d B i]+G_{R}[d B i]-P_{R}[d B m]
$$

$P_{T}$ represents the power of the transmitter and $G_{T}$ the gain of the transmitting antenna. $G_{R}$, the gain of the receiver antenna is $2.15 \mathrm{dBi}$, and $P_{R}$ is the value of the measured received signal strength [5]. The sum of the $P_{T}$ and $G_{T}$ values is the Equivalent Isotropically Radiated Power (EIRP), and is different in the three experiments due to hardware limitations. The exact EIRP is mentioned in each experiment description. An important concern is the speed of the sensing system consisting of WiFi nodes. Measurements and calculations are done quickly (less than $5 \mathrm{~ms}$ to compute the linear least squares algorithm). Only the execution time of the queries is slower because they have to go over the network, resulting in unpredictable response times in the order of maximum 300 $\mathrm{ms}$ to retrieve the resulting dataset of the sensors in this setup. With these numbers, one can conclude that a complete refresh of the path loss model can be done at least every second, which is more than satisfactory for the intended application. 


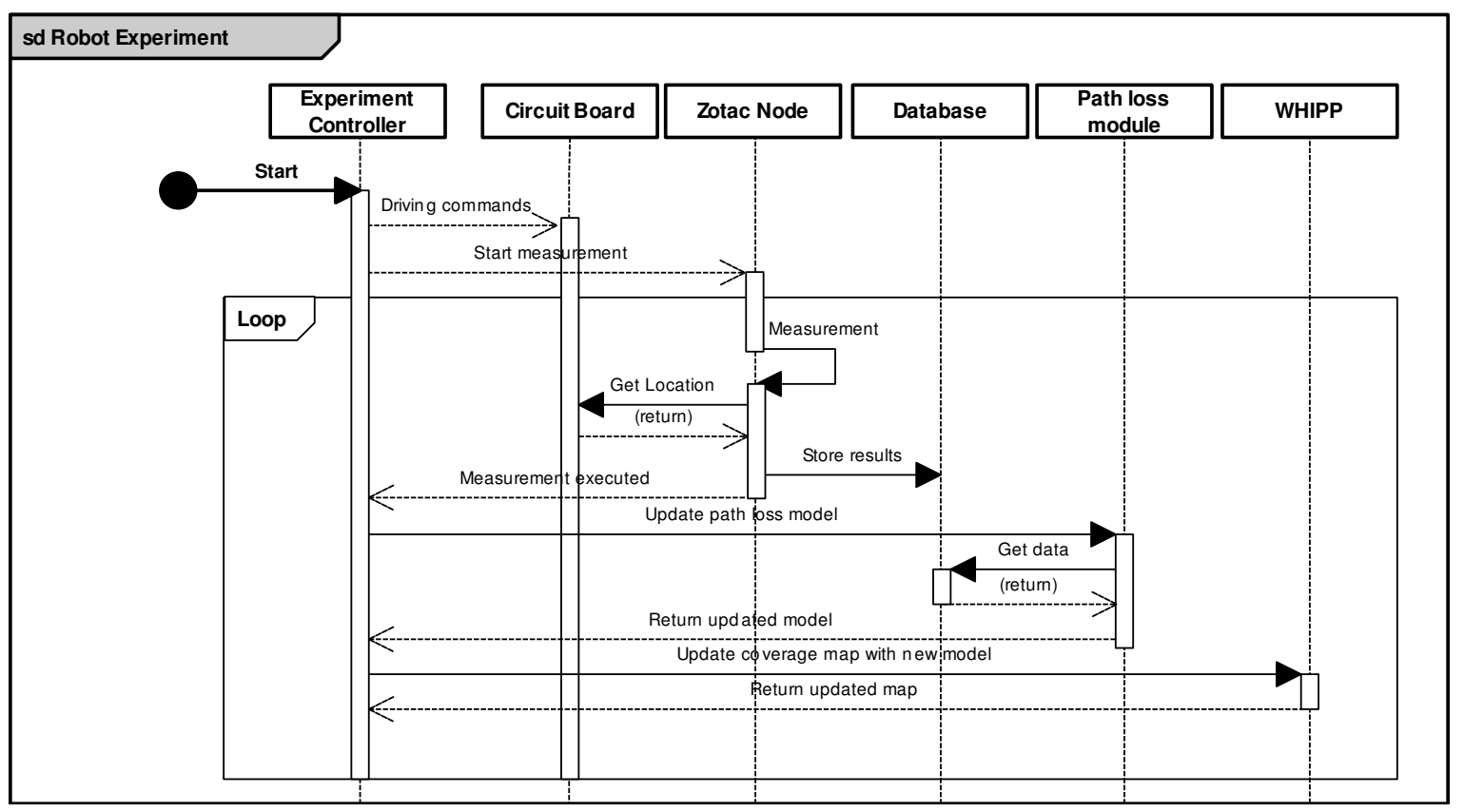

Fig. 6. Sequence diagram of an experiment

\section{EXPERIMENTS IN THE TESTBED}

In this paper, a set of experiments in the testbed is executed. All measurements are done in line of sight (LOS) of the measured access point.

\section{A. Fixed locations}

The first experiment is done with the fixed nodes in the testbed. Fig. 2 shows the set of nodes used for this experiment: 13 receiver nodes spread across the whole testbed, and 1 access point (node 22, indicated with two squares). This access point has been chosen at the far left side of the testbed, allowing to perform measurements over a bigger distance range $(6 \mathrm{~m}$ to $42.6 \mathrm{~m})$. The EIRP is set to the default value $(20 \mathrm{dBm})$ of the nodes in this experiment.

\section{B. Mobile measurements}

The second experiment is executed with a mobile robot that drives from the left to the right side of the testbed. The trajectory of the robot is shown in Fig. 2. The speed of the robot was set to $25 \mathrm{~cm} / \mathrm{s}$. In combination with the measurement interval of $1 \mathrm{~s}$, this results in a measurement sample every $25 \mathrm{~cm}$. The same access point is used as for the static experiment.

\section{Results}

The results of the measurements with the fixed and dynamic nodes are plotted in Fig. 7. The figure also shows the derived path loss models. These models look a bit different: the robot experiment resulted in a path loss model with a $2.47 \mathrm{~dB}$ lower $P L_{0}$ and 0.77 units lower $n$ in comparison with the static experiment. At a distance of $50 \mathrm{~m}$, the models already differ $15 \mathrm{~dB}$. For an explanation of these differences, we have to look at the architecture of the testbed: the fixed nodes are placed at a height of $2 \mathrm{~m}$, they are close to the top of the testbed where some metal pipes sometimes obstruct the LOS between the nodes. The metal itself also has some influence on the radiation pattern. The influence of these pipes on the robot measurements is certainly much smaller, due to its large separation from the ceiling. Since the height of the robot antenna is at $70 \mathrm{~cm}$, it is perfectly in LOS with the access point on every location, resulting in a lower path loss.

\section{EXPERIMENT IN AN OFFICE ENVIRONMENT}

\section{A. Measurements and path loss model}

A final experiment is conducted in the office environment described in Section II-A2. The robot followed a straight path through the corridor (indicated on Fig. 3) and performed a signal strength measurement every $10 \mathrm{~cm}$ along the path. The access point is indicated in Fig. 3 with a purple dot and its EIRP is set at $17 \mathrm{dBm}$.

The results are shown in Fig. 8. It is clear that the value of $P L_{0}$ differs significantly in comparison to the testbed parameters. This is valuable feedback for the planning tool, because now it can use these optimized parameters to calculate the coverage for this environment specifically.

\section{B. Feedback to the WHIPP tool}

As discussed before, the goal of the system is to integrate the measurements in the WHIPP tool. Every time a measurement is done, it is possible to update the coverage map of the environment with the new data. An example of such a generated map of the corridor, is shown in Fig. 3. The one slope path loss parameters are used as arguments for a coverage mapping algorithm that takes wall loss and 


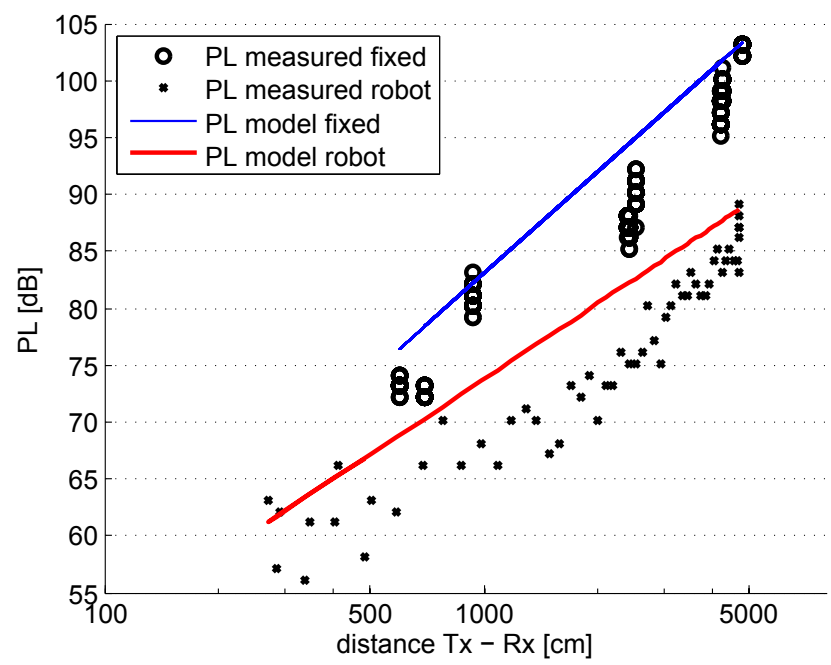

Fig. 7. Comparison of the path loss model constructed from measurements with fixed nodes at the testbed $\left(\mathrm{PL}_{0}=49.39 \mathrm{~dB}, \mathrm{n}=2.98\right.$ and $\left.\sigma=2.25 \mathrm{~dB}\right)$ to the model constructed with the robot $\left(\mathrm{PL}_{0}=46.92 \mathrm{~dB}, \mathrm{n}=2.21 . \sigma=2.78\right.$ $\mathrm{dB})$.

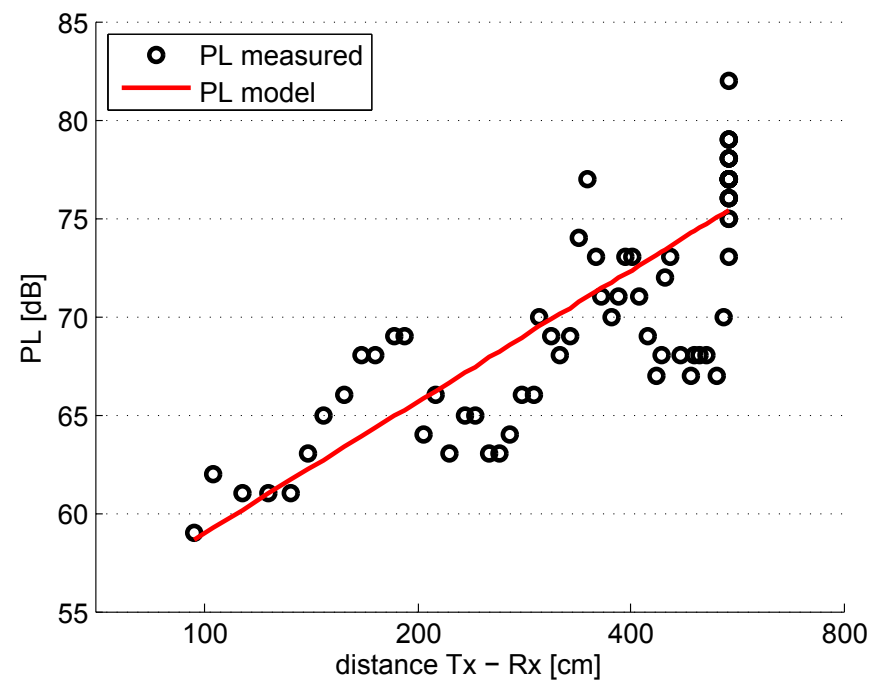

Fig. 8. Path loss model constructed from measurements with the robot in the office environment, $\mathrm{PL}_{0}=58.98 \mathrm{~dB}$ and $\mathrm{n}=2.21$. The standard deviation $\sigma$ is $3.13 \mathrm{~dB}$.

interaction loss into account [1], but as long as the location is within line of sight, the path loss function derived from the measurements is used as is.

\section{FUTURE WORK AND CONCLUSIONS}

In this paper, an automated system for path loss measurements has been made. A mobile robot was used to perform measurements. After each measurement, the path loss model of the environment was updated in real time. With the existing WHIPP tool, a coverage map of the area was created.
In the future, these measurements will play an important role in a cognitive networking system. They will be used to plan the ideal positions for access points. Once the network is deployed, the nodes will continuously monitor the environment and update the parameters of the access points in real time. This will enable us to fulfill the requirements of the wireless networks in an industrial environment.

\section{ACKNOWLEDGMENT}

This work was supported by the iMinds-ICON QoCON project, co-funded by iMinds, a research institute founded by the Flemish Government in 2004, and the involved companies and institutions.

\section{REFERENCES}

[1] Plets et al.: Coverage prediction and optimization algorithms for indoor environments. EURASIP Journal on Wireless Communications and Networking 2012 2012:123.

[2] E. Tanghe, W. Joseph, L. Verloock, Et al : The industrial indoor channel: large-scale and temporal fading at 900, 2400, and $5200 \mathrm{MHz}$., IEEE Trans. Wirel. Commun., 2008, 7, (7), pp. 27402751

[3] Crew project: Cognitive Radio Experimentation World, Internet: www.crew-project.eu/portal/wilab/open-environment-testbed-zwijnaarde

[4] Zotac ZBox, http://www.zotac.com/products/minipcs/zbox/product/zbox/category/mini-pcs/main-category/mini-pcs.html

[5] S. R. Saunders, Antennas and Propagation for Wireless Communication Systems Wiley and Sons, 1999.

[6] Wavemon network monitoring tool, http://freecode.com/projects/wavemon 